\title{
The Effects of Genetic Background for Diurnal Preference on Sleep Development in Early Childhood
}

This article was published in the following Dove Press journal: Nature and Science of Sleep

\author{
Isabel Morales-Muñoz, $\mathbb{D}^{1,2}$ \\ Katri Kantojärvi, ${ }^{1,3}$ Veli- \\ Matti Uhre, ' Outi Saarenpää- \\ Heikkilä, (ID ${ }^{4,5}$ Anneli Kylliäinen, ${ }^{6}$ \\ Pirjo Pölkki, ${ }^{7}$ Sari- \\ Leena Himanen, ${ }^{8,9}$ \\ Linnea Karlsson, ${ }^{10-12}$ \\ Hasse Karlsson, ${ }^{10-12}$ \\ E Juulia Paavonen, (D) ${ }^{1,13}$ \\ Tiina Paunio (D) ${ }^{1,3}$ \\ 'Department of Public Health Solutions, Finnish \\ Institute for Health and Welfare, Helsinki, Finland; \\ ${ }^{2}$ Institute for Mental Health, School of Psychology, \\ University of Birmingham, Birmingham, UK; \\ ${ }^{3}$ Department of Psychiatry and SleepWell \\ Research Program, Faculty of Medicine, University \\ of Helsinki and Helsinki University Central \\ Hospital, Helsinki, Finland; ${ }^{4}$ Pediatric Clinic, \\ Tampere University Hospital, Tampere, Finland; \\ ${ }^{5}$ Tampere Centre for Child Health Research, \\ University of Tampere and Tampere University \\ Hospital, Tampere, Finland; ${ }^{6}$ Psychology, Faculty of \\ Social Sciences, Tampere University, Tampere, \\ Finland; ${ }^{7}$ Department of Social Sciences, University \\ of Eastern Finland, Kuopio, Finland; ${ }^{8}$ Department of \\ Clinical Neurophysiology, Tampere University \\ Hospital, Medical Imaging Centre and Hospital \\ Pharmacy, Pirkanmaa Hospital District, Tampere, \\ Finland; ${ }^{9}$ Faculty of Medicine and Health \\ Technology, Tampere University, Tampere, Finland; \\ ${ }^{10}$ The FinnBrain Birth Cohort Study, Turku Brain \\ and Mind Center, Department of Clinical Medicine, \\ University of Turku, Turku, Finland; " Centre for \\ Population Health Research, Turku University \\ Hospital and University of Turku, Turku, Finland \\ ${ }^{12}$ Department of Psychiatry, University of Turku \\ and Turku University Hospital, Turku, Finland; \\ ${ }^{13}$ Pediatric Research Center, Child Psychiatry, \\ University of Helsinki and Helsinki University \\ Hospital, Helsinki, Finland
}

Correspondence: Isabel Morales-Muñoz Tel +358295246000

Email isabel.morales@thl.fi

Tiina Paunio

Tel +35829410294l

Email tiina.paunio@helsinki.fi
Purpose: No previous research has examined the impact of the genetic background of diurnal preference on children's sleep. Here, we examined the effects of genetic risk score for the liability of diurnal preference on sleep development in early childhood in two population-based cohorts from Finland.

Participants and Methods: The primary sample (CHILD-SLEEP, CS) comprised 1420 infants (695 girls), and the replication sample (FinnBrain, FB; 962 girls) 2063 infants. Parent-reported sleep duration, sleep-onset latency and bedtime were assessed at three, eight, 18 and 24 months in CS, and at six, 12 and 24 months in FB. Actigraphy-based sleep latency and efficiency were measured in CS in 365 infants at eight months (168 girls), and in 197 infants at 24 months ( 82 girls). Mean standard scores for each sleep domain were calculated in both samples. Polygenic risk scores (PRS) were used to quantitate the genetic risk for eveningness (PRSBestFit) and morningness (PRS10kBest).

Results: PRSBestFit associated with longer sleep-onset latency and later bedtime, and PRS10kBest related to shorter sleep-onset latency in CS. The link between genetic risk for diurnal preference and sleep-onset latency was replicated in $\mathrm{FB}$, and meta-analysis resulted in associations $(\mathrm{P}<0.0005)$ with both PRS-values (PRSBestFit: $\mathrm{Z}=3.55$; and PRS10kBest: $\mathrm{Z}=$ -3.68). Finally, PRSBestFit was related to actigraphy-based lower sleep efficiency and longer sleep latency at eight months.

Conclusion: Genetic liability to diurnal preference for eveningness relates to longer sleeponset during the first two years of life, and to objectively measured lowered sleep efficiency. These findings enhance our understanding on the biological factors affecting sleep development, and contribute to clarify the physiological sleep architecture in early childhood.

Keywords: genetic risk, chronotype, sleep, early childhood, cohorts

\section{Introduction}

Circadian rhythms are cyclical changes in cellular, molecular, and biological processes repeating approximately every 24 hours. ${ }^{1}$ They are driven by an internal "master clock", and play a central role in the regulation of many physiological processes, including sleep-wake cycles. ${ }^{2}$ Chronotype, or diurnal preference, refers to the individual timing of the endogenous circadian system affecting the preferred behavioral patterns including timing of sleep and wake. ${ }^{3}$ Chronotype falls on a continuum with individuals in one extreme who are referred to as morning types, and individuals in the other extreme, being referred to as evening types.

Circadian rhythmicity has a genetic basis ${ }^{4}$ and the genetic mechanisms of the core circadian clock has been also established. ${ }^{5}$ Consistently, inter-individual differences 
in chronotype are also determined by genetic factors, ${ }^{1}$ although age, gender and environmental light exposure may also explain this variation. For example, twin and family studies have estimated the heritability of chronotype to be up to $50 \% .^{6-8}$ Furthermore, recent genome-wide association studies (GWAS) have identified specific genetic variations associated with self-reported chronotype. ${ }^{9-12}$ Based on these studies, one can calculate quantitative polygenic risk scores (PRS), which can be used for estimation of individual genetic proneness for diurnal preference.

In adolescents and adults, evening chronotype associates with negative social and health consequences. ${ }^{13,14}$ Furthermore, chronotype influences sleep quality. ${ }^{15-17}$ However, little is known about its effect on sleep-wake patterns in early childhood. The scarce existing studies indicate that parent-reported morningness in toddlers associates with earlier bedtimes, sleep onset times, and wake times, ${ }^{18}$ while evening types show bedtime resistance, and wake in a negative mood. ${ }^{19}$ Further, we recently reported that self-reported maternal eveningness associated with several sleep problems in children from three to 24 months of age. ${ }^{20}$

Examining chronotype-sleep association is relevant in early childhood because this stage is characterized by substantial inter-individual differences in sleep patterns. $^{21,22}$ Furthermore, a high percentage of parents report their children experiencing sleep difficulties during the first two years of life. ${ }^{22,23}$ Therefore, a better understanding of the factors affecting sleep development is needed. However, all the previous studies in children have only used parent-reported measures of chronotype, while none have utilized data about the genetic factors of chronotype, such as the polygenic risk estimates.

The main objective of this study was to examine whether PRS for eveningness (http://www.nealelab.is/blog/2017/7/19/ rapid-gwas-of-thousands-of-phenotypes-for-337000-samplesin-the-uk-biobank) and morningness, ${ }^{12}$ are related to parentreported nighttime and daytime sleep duration, sleep-onset latency and bedtime during the first two years of life, in two population-based birth cohorts. We selected these sleep variables because our main interest was to investigate those sleep variables that assumingly would be related to circadian preference, ie bedtime, sleep onset latency and sleep duration. ${ }^{20}$ They present a high variability among infants in early childhood, ${ }^{22}$ and this high variability is likely to be related to several influencing variables, such as genetic factors, and in this case, polygenic risk scores for chronotype. Further, we also examined whether differences between boys and girls exist, and how the genetic risk for diurnal preference manifests in sleep at different stages of early childhood. Finally, we studied the relation of the genetic risk scores on actigraphybased sleep measures. We hypothesized that PRS for eveningness would relate to shorter sleep duration, longer sleep-onset latency and later bedtime, ${ }^{20}$ while PRS for morningness would associate with longer sleep duration, shorter sleep-onset latency and earlier bedtime. ${ }^{18}$

\section{Participants and Methods Participants}

The primary sample of this study, CHILD-SLEEP (CS), is a birth cohort in Pirkanmaa, southern Finland. ${ }^{24}$ For this study, we used parental questionnaires during pregnancy (32nd week), umbilical cord blood samples at birth, questionnaire-based sleep measures of the infants at three, eight, 18 and 24 months, and actigraphy-based sleep measures at eight and 24 months. The initial dataset comprised 1679 families. Further, 14 children with chronic illnesses were excluded. From this initial sample, 1420 umbilical cord blood samples were available. Of these, we had questionnaire data from $99.2 \%(\mathrm{~N}=1409)$ infants at three months, $90.3 \%$ $(\mathrm{N}=1282)$ at eight months, $80.6 \%(\mathrm{~N}=1145)$ at 18 months, and $65.8 \%(\mathrm{~N}=935)$ at 24 months. While all the questionnaires and umbilical blood samples were obtained from the whole sample, actigraphy measures were only conducted in 365 cases at eight months, and 197 at 24 months.

The replication sample of this study consists of another population-based cohort, the FinnBrain (FB), in southwest Finland (www.finnbrain.fi). ${ }^{25}$ In all, 5790 out of 8895 women visiting the recruitment sites during pregnancy (12th week) were contacted. A total of 3808 (66\%) mothers decided to participate. Umbilical blood sample were obtained from 2063 infants, and 10 cases were excluded due to reported chronic illnesses. We finally included only participants with parent-reported sleep data available, ie 1491 infants at six months, 1214at 12 months and 994 at 24 months. In this case, actigraphy-based sleep measures were not available.

\section{Ethics Statement}

The CS cohort was reviewed and approved by the Pirkanmaa Hospital District Ethical Committee (9/3/2011, Ethical Research Permission Code R11032), and the FB cohort by the Ethical Committee of the Southwestern Finland Hospital District (number 57/180/2011). Permission for the recruitment procedure in both cohorts was received from the physicians at 
the target health centers. Parental informed consent was obtained for all participants, in accordance with the Declaration of Helsinki.

\section{Measures}

\section{Genetic Estimates for Chronotype}

In both cohorts, DNA samples were extracted according to standard procedures at the Finnish Institute for Health and Welfare. DNA samples for CS were genotyped with Illumina Infinium PsychArray and for FB with Illumina Infinium PsychArray and Illumina Infinium Global Screening Array at Estonian Genome Centre. Quality control was performed with PLINK 1.9 (www.cog-genomics.org/plink/1.9/). ${ }^{26}$ Markers were removed for missingness $(>5 \%)$ and Hardy-Weinberg equilibrium ( $\mathrm{p}$-value $<1 \times 10-6$ ). Individuals were checked for missing genotypes $(>5 \%)$, relatedness (identical by descent calculation, PI_HAT $>0.2$ ) and population stratification (multidimensional scaling). Principal component analysis (PCA) for population stratification was calculated with PLINK 1.9. Genotyped data in CS was imputed with SHAPEITv2 ${ }^{27}$ and IMPUTEv $2^{28}$ against Finnish WGS and 1000 genomes reference panels. Genotyped data of FB was pre-phased with Eagle v2.4 ${ }^{29}$ and imputed with Beagle 4.1. ${ }^{30}$ SISU-project wholegenome sequencing data was used as imputation reference panel.

PRS $_{\text {BestFit }}$ was derived from UK Biobank sample consisting of 301,143 individuals (UK Biobank GWAS Manifest 20,170,915, phenotype code: 1180; http://www.nealelab.is/ blog/2017/7/19/rapid-gwas-of-thousands-of-phenotypes-for -337000-samples-in-the-uk-biobank). In UK Biobank sample phenotype for eveningness was estimated with the question "Do you consider yourself to be?" "Definitely a 'morning' person, More a 'morning' than an 'evening' person, More an 'evening' than a 'morning' person or Definitely an 'evening' person". We tested the best fit p-value threshold for eveningness with a national sample of FINRISK where information about the chronotype was collected with the same question as in UK Biobank study. Best fit $\mathrm{p}$-value threshold was 0.007 . This was used as a measure of eveningness. We used PRSice program ${ }^{31}$ to estimate PRS for studied individuals. PRS $_{10 \mathrm{kBest}}$ was derived from genome-wide association studies (GWAS) from 697,828 UK Biobank and 23 andMe, ${ }^{12}$ which was treated as a measure of morningness. In 23andMe cohort, phenotype for morningness was estimated with two identical questions ("Are you naturally a night person or a morning person?"). For the first instance, the possible answers were "Night owl", "Early bird" and "Neither", and for the second "Night person",
"Morning person", "Neither", "It depends" and "I'm not sure". Individuals with discordant or neutral responses to both were excluded. 10,000 most significant SNPs were included in $\mathrm{PRS}_{10 \mathrm{kBest}}$. Detailed description of this procedure has been described elsewhere. ${ }^{12}$ Correlations between $\mathrm{PRS}_{\mathrm{BestFit}}$ and $\mathrm{PRS}_{10 \mathrm{kBest}}$ showed moderate associations in $\mathrm{CS}(\mathrm{r}=-0.361, \mathrm{p}<0.001)$ and $\mathrm{FB}(\mathrm{r}=-0.314, \mathrm{p}<0.001)$.

\section{Subjective Sleep Measures}

The Brief Infant Sleep Questionnaire (BISQ) was used to evaluate infant sleep quality. ${ }^{32}$ We selected the following items: (i) nocturnal sleep hours; (ii) daytime sleep hours; (iii) sleep-onset latency; and (iv) bedtime. BISQ was used in $\mathrm{CS}$ at all time points. Furthermore, sleep-onset latency and bedtime were also obtained in FB, at six and 12 months. The four sleep items were based on open-ended items.

The Infant Sleep Questionnaire (ISQ) assesses infant sleeping habits on an interval scale with seven to nine response alternatives. ${ }^{33}$ For this study, sleep-onset latency at all time points in CS cohort was selected, as follows: How long does it usually take to settle your baby off to sleep on average?: $1=<10$ minutes; $2=10-20$ minutes; $3=20-30$ minutes; $4=30-40$ minutes; $5=40-50$ minutes; $6=50-60$ minutes; or $7=\geq 1$ hour. We additionally included the ISQ sleep-onset latency item in this study to control for potential subjective bias associated with open-ended items (ie BISQ items), which can result in data with differential quality. ${ }^{34}$

The Sleep Disturbance Scale for Children (SDSC) assesses the frequency of a variety of sleep-related behaviors in preschoolers, ${ }^{35}$ children and adolescents, ${ }^{36}$ as follows: "How long after going to bed does your child usually fall asleep: $1=<15$ minutes; $2=15-30$ minutes; $3=30-45$ minutes; 4=45-60 minutes; or 5 $\leq 60$ minutes." This scale was used to measure sleep-onset latency at 24 months in FB cohort.

The World Health Organization (WHO)-coordinated survey sleep items are derived from the school children's health behavior study. ${ }^{37}$ In FB, parents were asked to report about their child bedtime at the age of 24 months, as follows: "When does your child usually go to bed?" There were alternatives ranging from 21:00 to 02:00 hours or later, in half hour intervals.

\section{Objective Sleep Measures}

For this study, Actiwatch 7 was used (CamNtech Ltd, Cambridge, United Kingdom). Parents were asked to place the actigraphy on the thigh of their infant for three consecutive days (ie two nights) and to complete the sleep$\log$. Nighttime activity data were scored using the sleep 
analysis program provided by the manufacturer. This device uses the Oakley algorithm, ${ }^{38}$ which has been previously validated in infants. ${ }^{39}$ Further, we used a medium sensitivity (wake threshold), following previous studies using similar devices in pediatric population. ${ }^{40}$ All cases with only one night recorded $(n=10)$ were excluded from the final analyses. Our sleep variables of interest were sleep efficiency and sleep latency.

\section{Covariates}

The following covariates were considered: age (days), sex, three top principal components to adjust the effect estimates for population stratification and season of assessment. Seasons were defined in terms of the amount of daily light at the date of assessment: (i) $\mathrm{IL}=$ increasing light: Feb 5-May 5; (ii) L= light: May 6-Aug 5; (iii) $\mathrm{ID}=$ increasing darkness: Aug 6-Nov 6; and (iv) $\mathrm{D}=$ dark: Nov 7-Feb 4.

Sociodemographic variables and description of the variables of interest appear in Table 1.

\section{Statistical Analyses}

Statistical analyses were performed with SPSS V25.0. The mean standard scores for the main outcome (ie each sleep domain) were calculated by summing the standard score of each time point from one specific sleep domain and dividing by four, which was the number of time points. By using this approach, instead of other longitudinal analysis approaches, such as Latent Growth Curve Analysis, we assume that we are missing several cases, due to attrition, across the time points. However, and taking into account the high variability inter- and intra-subject in sleep development that is observed in early childhood, the approach presented here is the most suitable one for the purpose of this study.

To test our primary hypothesis that genetic risk for diurnal preference is related to sleep, linear regression models were conducted in CS. To do this, mean standard scores for each sleep domain were used as dependent variables, while $\mathrm{PRS}_{\text {BestFit }}$ and $\mathrm{PRS}_{10 \mathrm{kBest}}$ were used as independent variables. Each independent and dependent variable were conducted in different models, together with all the covariates. The acquired $P$ values were adjusted to control false discovery rate (FDR) using the Benjamini-Hochberg procedure. ${ }^{41}$

Similar regression models, using mean standard scores were applied in FB cohort. Meta-analyses using the meta data from the CS and FB cohorts were performed using R $3.60^{42}$ with "meta" package using fixed effect model. ${ }^{43}$
Table I Socio-Demographic Factors and Variables of Interest in CHILD-SLEEP and FinnBrain

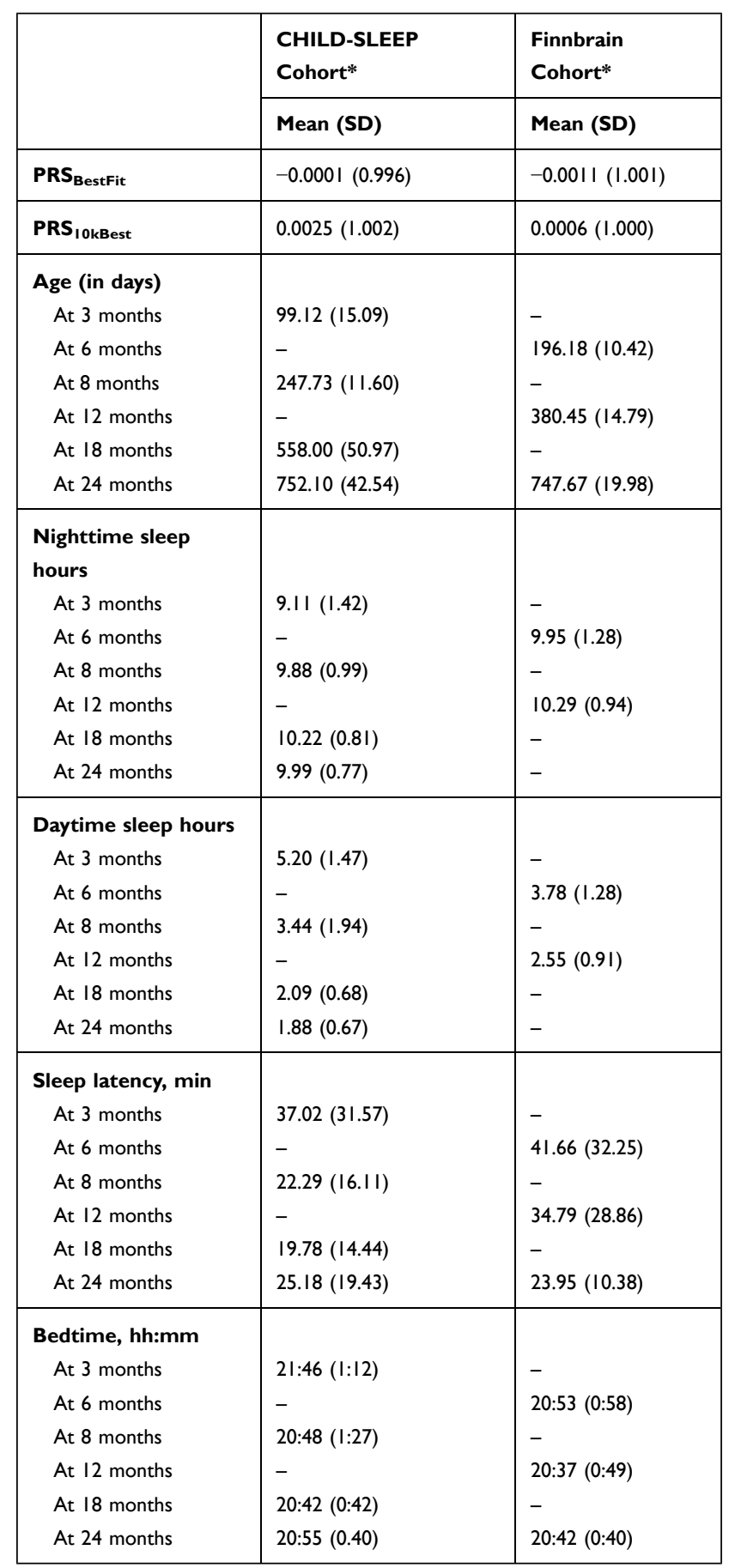

Note: *CHILD-SLEEP cohort sample in this study comprised 695 girls (47.2\%) and 778 boys (52.8); and FinnBrain cohort sample 962 girls (47.0\%) and 1083 boys (53.0\%).

Abbreviations: PRS $_{\text {Bestfit, }}$ Polygenetic risk score for eveningness; PRS $_{\text {IOkBest, }}$ Polygenetic risk score for morningness.

To examine whether differences between boys and girls appeared in CS, the interaction terms between sex and PRS were added in a secondary analysis. Furthermore, to examine how the genetic risk for diurnal preference expresses at 
different time points, separate linear regression models were applied, at each time point in both cohorts. Finally, linear regression models were applied to examine the links between genetic risk for diurnal preference and actigraphybased sleep measures in CS; first, using mean standard scores for each actigraphy domain; and second, using separate regression analyses at eight and 24 months.

\section{Results}

\section{Genetic Risk for Diurnal Preference and Parent-Reported Sleep in CS}

$\mathrm{PRS}_{\text {BestFit }}$ associated with mean standard scores of longer sleep-onset latency using both BISQ and ISQ $\left(\mathrm{R}^{2}=0.019\right.$, $\beta=0.081, \quad p=0.004 ;$ and $R^{2}=0.020, \quad \beta=0.081, \quad p=0.004$, respectively). Additionally, $\mathrm{PRS}_{\mathrm{BestFit}}$ associated with mean standard scores of later bedtime $\left(\mathrm{R}^{2}=0.028\right.$, $\beta=0.085, p=0.003)$. PRS $10 \mathrm{kBest}$ associated with mean standard scores of shorter ISQ sleep-onset latency $\left(R^{2}=0.016\right.$, $\beta=-0.060, p=0.034)$.

After Benjamini-Hochberg multiple testing correction, the associations between $\mathrm{PRS}_{\mathrm{BestFit}}$ and longer BISQ and ISQ sleep-onset latency, as well as $\mathrm{PRS}_{\text {BestFit }}$ and bedtime remained significant (FDR $<0.005$; see Table 2). For description of the mean values and standard errors of ISQ sleep-onset latency for each quartile of $\mathrm{PRS}_{\text {BestFit }}$ and PRS $\mathrm{PRkBest}_{10 \mathrm{~s}}$, see Figure 1.

\section{Replication in FB}

We aimed at replication of the significant findings (ie association of $\mathrm{PRS}_{\mathrm{BestFit}}$ and $\mathrm{PRS}_{10 \mathrm{kBest}}$ with sleep-onset latency and bedtime) in FB. The results only showed association between $\mathrm{PRS}_{10 \mathrm{kBest}}$ and BISQ sleep-onset latency $\left(\mathrm{R}^{2}=0.014, \beta=-0.049, \mathrm{p}=0.034\right)$. However, no significant associations were observed between any of the PRSes and bedtime (see supplementary Table 1).

\section{Meta-Analysis}

Mean standard score regarding BISQ sleep-onset latency was significantly associated with both genetic scores $\left(\mathrm{PRS}_{\mathrm{BestFit}}\right.$, $\mathrm{Z}=3.55, \quad \mathrm{p}=0.0004 ; \quad \mathrm{PRS}_{10 \mathrm{kBest}}, \quad \mathrm{Z}=-3.68, \quad \mathrm{p}=0.0002$ ). Combining BISQ sleep-onset latency from FB-cohort and ISQ sleep-onset latency from CS-cohort strengthened this association $\left(\mathrm{PRS}_{\mathrm{BestFit}}: \mathrm{Z}=3.62, \mathrm{p}=0.0003\right.$ and $\mathrm{PRS}_{10 \mathrm{kBest}}$ : $\mathrm{Z}=-4.11, \mathrm{P}<0.0001$ ) (see Figure 2).

\section{Differences Between Boys and Girls in CS}

No significant interactions of gender with any of the PRS values were observed in any of the associations.

\section{Associations Between Genetic Risk for Diurnal Preference and Parent-Reported Sleep-Onset Latency at Separate Time Points}

To study the temporal pattern of manifestation of genetic risk for diurnal preference on sleep-onset latency, the most consistent result in both cohorts, we analyzed this sleep variable separately at each time point (see supplementary Table 2). As sleep development is highly variable at different stages of early childhood, ${ }^{22}$ we wanted to investigate whether the effect of PRS for chronotype in sleep-onset latency was more relevant at a specific stage of the child's development.

Results from CS indicated that both $\mathrm{PRS}_{\mathrm{BestFit}}$ and $\mathrm{PRS}_{10 \mathrm{kBest}}$ were associated with ISQ sleep-onset latency at three $\left(\mathrm{R}^{2}=0.022, \beta=0.081, \mathrm{p}=0.007\right.$; and $\mathrm{R}^{2}=0.021, \beta=$ $-0.077, \mathrm{p}=0.008$, respectively) and 18 months $\left(\mathrm{R}^{2}=0.020\right.$, $\beta=0.087, p=0.009 ;$ and $R^{2}=0.018, \beta=-0.066, p=0.037$, respectively), and with BISQ sleep-onset latency at 18

Table 2 Associations Between Genetic Risk for Diurnal Preference and Mean Standard Scores for Each Sleep Domain, in CHILDSLEEP

\begin{tabular}{|l|l|l|l|l|l|l|l|}
\hline CHILD-SLEEP Cohort & \multicolumn{3}{|l|}{ PRS } & \multicolumn{2}{l|}{ PESTFIT } & \multicolumn{2}{l|}{ PRS I OKBEST } \\
\hline Sleep Domains & $\mathbf{N}$ & $\mathbf{R}^{\mathbf{2}}$ & $\boldsymbol{\beta}$ & $\mathbf{P}$ & $\mathbf{R}^{\mathbf{2}}$ & $\boldsymbol{\beta}$ & $\mathbf{P}$ \\
Night sleep duration & 1262 & 0.011 & -0.033 & 0.253 & 0.012 & 0.035 & 0.210 \\
Daytime sleep duration & 1262 & 0.008 & 0.053 & 0.109 & 0.005 & -0.081 & 0.936 \\
ISQ Sleep-onset latency & 1262 & $\mathbf{0 . 0 1 9}$ & $\mathbf{0 . 0 8 1}$ & $\mathbf{0 . 0 0 4} * *$ & $\mathbf{0 . 0 1 6}$ & $-\mathbf{0 . 0 6 0}$ & $\mathbf{0 . 0 3 4}$ \\
BISQ Sleep-onset latency & 1262 & $\mathbf{0 . 0 2 0}$ & $\mathbf{0 . 0 8 1}$ & $\mathbf{0 . 0 0 4} * *$ & 0.016 & -0.045 \\
Bedtime & 1262 & $\mathbf{0 . 0 2 8}$ & $\mathbf{0 . 0 8 5}$ & $\mathbf{0 . 0 0 3 * *}$ & 0.021 & 0.115 \\
\hline
\end{tabular}

Notes: Bold indicates statistically significant associations $(\mathrm{P}<0.05)$.**After FDR correction, the associations between PRS bedtime remained significant (FDR<0.005).

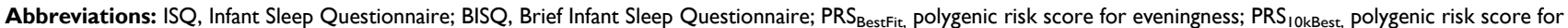
morningness. 

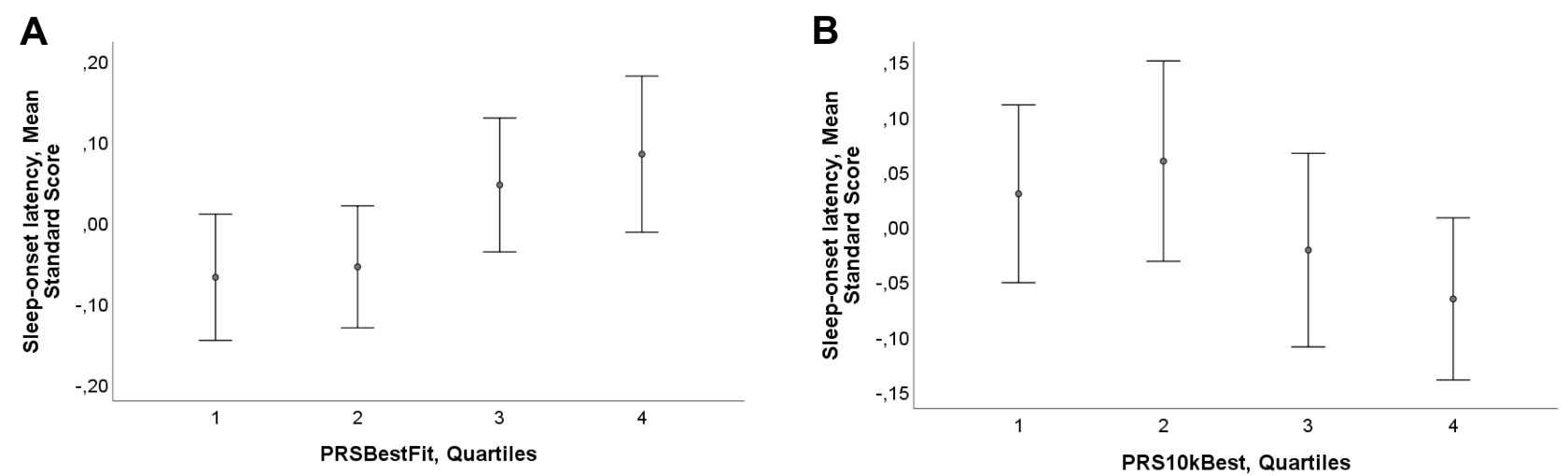

Figure I Sleep-onset latency and genetic risk for diurnal preference in early childhood. This graph presents the mean and standard error (SE) in parent-reported sleep-

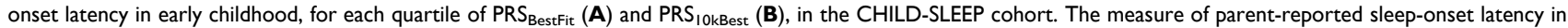
childhood refers to mean standard scores at three, eight, 18 and 24 months, from the Infant Sleep Questionnaire. Graph A indicates that those subjects situated in the 4th Quartile (ie higher PRS for eveningness) report higher mean standard score values in ISQ sleep-onset latency (ie longer sleep-onset latency). Accordingly, graph B shows that those infants located in the 4th Quartile (ie higher PRS for morningness) obtain lower mean standard values in ISQ sleep-onset latency (ie short sleep-onset latency). Error bars represent $2 \mathrm{SE}$, indicating $95 \%$ of values that are less than two standard errors (2SE) away from the mean.
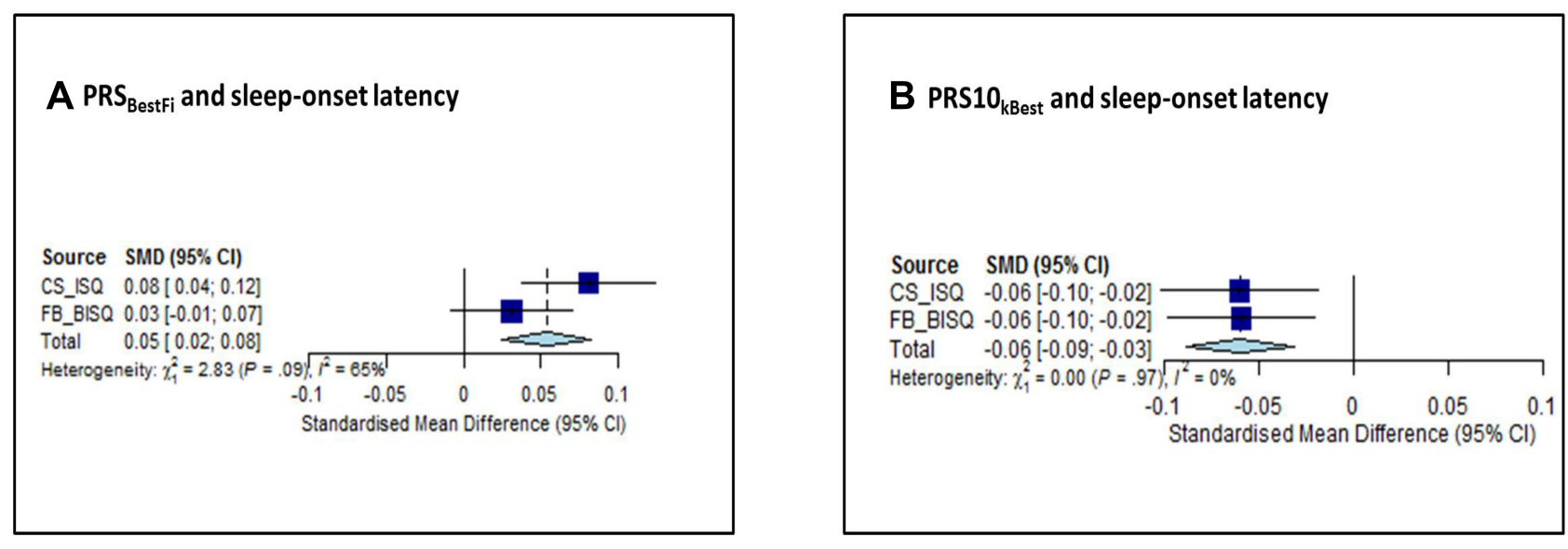

Figure 2 Forest plot from the meta-analysis, concerning genetic risk for diurnal preference and sleep-onset latency in early childhood. This graph provides a graphical display of the estimated results when we combined the data from both cohorts (CS and FB) to perform meta-analysis. Graph (A) represents the forest plot from the meta-analysis, concerning the association between $\mathrm{PRS}_{\text {Bestrit }}$ and sleep-onset latency in early childhood. Graph (B) represents the forest plot from the meta-analysis, concerning the association between PRS ${ }_{\text {IokBest }}$ and sleep-onset latency in early childhood. First dark blue square on the top, in each graph, represents the standard mean differences (and 95\% Confidence Intervals) for sleep-onset latency measured with Infant Sleep Questionnaire in CS, and the dark blue square below, in each graph, refers to the standard mean differences (and 95\% Confidence Intervals) for sleep-onset latency measured with Brief Infant Sleep Questionnaire in FB. The light blue diamond on the bottom appearing in each graph represents the overall effect estimate.

Abbreviations: SMD, standardized mean difference; Cl, confidence interval; CS, CHILD-SLEEP; FB, FinnBrain; ISQ, Infant Sleep Questionnaire; BISQ, Brief Infant Sleep Questionnaire; PRSBestFit, polygenic risk score for eveningness; PRSIOkBest, polygenic risk score for morningness.

months $\left(\mathrm{R}^{2}=0.028, \beta=0.096, \mathrm{p}=0.005\right.$; and $\mathrm{R}^{2}=0.025, \beta=$ $-0.073, \mathrm{p}=0.026$, respectively). Further, PRS $\mathrm{BestFit}_{\text {asso- }}$ ciated with BISQ sleep-onset latency at three months $\left(\mathrm{R}^{2}=0.028, \beta=0.087, \mathrm{p}=0.005\right)$, and $\mathrm{PRS}_{10 \mathrm{kBest}}$ related to ISQ sleep-onset latency at 24 months $\left(R^{2}=0.025, \beta=\right.$ $-0.069, p=0.050$ ). Interestingly, no significant associations with sleep-onset latency at eight months were observed. To further understand this lack of association, we conducted again this analysis in those children with actigraphy at eight months, and this new analysis confirmed this lack of association (see supplementary Table 3).
In $\mathrm{FB}$, we found associations between $\mathrm{PRS}_{10 \mathrm{kBest}}$ and BISQ sleep-onset latency at 12 months $\left(\mathrm{R}^{2}=0.017, \beta=\right.$ $-0.061, \mathrm{p}=0.035$ ), while no associations at $\mathrm{P}<0.05$ were found with $\mathrm{PRS}_{\text {BestFit. }}$

\section{Genetic Risk for Diurnal Preference and Actigraphy-Based Sleep Measures in CS}

There was significant association between $\mathrm{PRS}_{\text {BestFit }}$ and the mean standard score for actigraphy-based sleep efficiency $\left(R^{2}=0.054, \beta=-0.150, p=0.008\right)$. In separate analysis of the time points, there were significant relations between $\mathrm{PRS}_{\mathrm{BestFit}}$ 
and sleep efficiency $\left(\mathrm{R}^{2}=0.055, \beta=-0,154, \mathrm{p}=0.008\right)$ and between $\mathrm{PRS}_{\text {BestFit }}$ and sleep latency $\left(\mathrm{R}^{2}=0.045, \beta=0.116\right.$, $\mathrm{p}=0.046$ ) at eight months. Finally, no significant associations were found for $\mathrm{PRS}_{10 \mathrm{kBest}}$ (see Table 3).

\section{Discussion}

Our main findings indicate that genetic liability to eveningness associates with parent-reported longer sleep-onset latency and later bedtime in early childhood, while preference for morningness relates to shorter sleep-onset latency. This association between genetic risk for parentreported diurnal preference and parent-reported sleeponset latency was replicated in another birth cohort in Finland. Finally, we found that the polygenic background for eveningness related to longer actigraph-based sleep latency at eight months and lower sleep efficiency in early childhood.

Our findings are consistent with previous questionnaire-based studies, where toddlers exhibiting parentreported morningness had earlier sleep onset times. ${ }^{18}$ We found that both PRS for eveningness and morningness were associated with longer and shorter parent-reported sleep-onset latency in early childhood, respectively; and PRS for eveningness was also linked to longer actigraphybased sleep latency at eight months. These findings are also in line with our previous studies, where we reported self-reported maternal eveningness being related to longer sleep-onset latency at three, 18 and 24 months. ${ }^{20}$ Further, our current findings are also consistent with previous studies in adolescent twins showing that the influence of genetic factors was greatest for actigraphy-based sleeponset latency. ${ }^{44}$

Secondly, we found associations between PRS for eveningness and later bedtime during early childhood. These findings support previous studies showing that self- reported maternal eveningness ${ }^{20}$ associated with later bedtimes in early childhood. Further, previous research has shown that parent-reported morningness in toddlers concurrently associated with earlier bedtimes. ${ }^{18}$ In our study, we did not observe significant associations between PRS for morningness and earlier bedtime. This might be explained by the fact that our sample was considerably younger than previous research using parent-reported chronotype in toddlers.

Thirdly, when we examined the associations between genetic risk for diurnal preference and parent-reported sleep-onset latency at different time points, we found that PRS for eveningness related to sleep-onset latency at three and 18 months, while no associations were found at eight months or 24 months. This suggests that the genetic contribution of chronotype may be masked by co-occurring environmental and developmental factors, challenging the parental evaluation of sleep parameters at these specific stages. This is supported in our finding that PRS for eveningness associated with sleep-onset latency in eight-month -old infants when measured with actigraphy. It could be that subjective evaluation of sleep onset latency is challenged in specific time periods like shown in findings of discrepancies ${ }^{45}$ and underreports ${ }^{46}$ of sleep onset times by parents of preschoolers compared to actigraphy measures.

Interestingly, we did not find association between genetic risk for diurnal preference and sleep duration in early childhood. These results contradict previous research using parent-reported chronotype. ${ }^{20,47}$ However, this lack of association is consistent with previous research conducted in young adult twins ${ }^{6}$ and school-aged children, ${ }^{48,49}$ as well as with a large study in adult population showing non-significant genetic association between sleep duration and chronotype ${ }^{50}$ Furthermore, previous findings from 18 month-old twins support the idea that

Table 3 Associations Between Genetic Risk for Diurnal Preference and Actigraphy-Based Sleep Variables, in CHILD-SLEEP

\begin{tabular}{|c|c|c|c|c|c|c|c|c|}
\hline & \multirow[b]{2}{*}{ Time Point } & \multirow[b]{2}{*}{$\mathbf{N}$} & \multicolumn{3}{|c|}{ PRS $_{\text {BestFit }}$} & \multicolumn{3}{|c|}{ PRS $_{\text {IOkBest }}$} \\
\hline & & & $\mathbf{R}^{2}$ & $\boldsymbol{\beta}$ & $\mathbf{p}$ & $\mathbf{R}^{2}$ & $\beta$ & $\mathbf{p}$ \\
\hline \multirow[t]{3}{*}{ Night sleep efficiency } & Mean standard scores & 320 & 0.054 & -0.150 & 0.008 & 0.036 & 0.058 & 0.307 \\
\hline & 8 months & 300 & 0.055 & -0.154 & 0.008 & 0.035 & 0.049 & 0.399 \\
\hline & 24 months & $13 \mid$ & 0.049 & -0.035 & 0.698 & 0.052 & -0.068 & 0.459 \\
\hline \multirow[t]{3}{*}{ Night sleep latency } & Mean standard scores & 300 & 0.018 & 0.062 & 0.280 & 0.015 & -0.018 & 0.752 \\
\hline & 8 months & 320 & 0.045 & 0.116 & 0.046 & 0.032 & 0.009 & 0.874 \\
\hline & 24 months & $|3|$ & 0.049 & -0.140 & 0.127 & 0.032 & 0.042 & 0.652 \\
\hline
\end{tabular}

Note: Bold indicates statistically significant associations $(p<0.05)$.

Abbreviations: PRS $_{\text {BestFit, }}$ polygenic risk score for eveningness; PRS $_{10 k B e s t,}$ polygenic risk score for morningness. 
short sleep may be mainly explained by common shared environmental factors. ${ }^{51}$

This study has some limitations. First, the amount of bright light during the day could contribute to individual differences in sleep difficulties. ${ }^{52}$ However, we controlled for season of assessment, based on the changes in the amount of daily light. Second, there are some other confounding factors that might influence our results, such as environmental factors (ie daycare, especially at 18 and 24 months) or children's developmental factors. Further, it is important to take into account the important role of environmental factors, in conjunction with genetic ones, when investigating the influences on sleep traits in early childhood. In fact, this is something that we have already reported in our previous research on the effects of parental chronotype ${ }^{20}$ and maternal risk factors ${ }^{53}$ in the development of sleep problems in early childhood. In this current study, we were interested in reporting the child's genetic contribution (ie PRS for diurnal preference), but these findings should be interpreted with caution, as relevant parent-related environmental factors might also exert some influence. Third, as is usual in birth cohort studies, we experienced loss of study cases across the time points. However, the attrition rate in our study was relatively low, which ensures the validity of the findings. Fourth, although we reported significant associations between genetic liability and subjective sleep pattern (ie parent-reported sleep-onset latency and bedtime), the effect size of these associations were relatively small; however, the effect size between genetic liability and objective sleep measures (ie actigraph-based sleep latency and sleep efficiency) was considerably bigger. Therefore, this suggests that when examining the associations between genetic liability and parent-reported sleep development in early childhood, several contributing variables, probably associated with parentrelated factors, might also play an important role in these associations. However, the bigger effect size observed with the actigraphy indicates that these associations appear stronger when subjective factors are controlled. Fifth, some other sleep timing parameters of interest could have also been considered for this study, such as midpoint of sleep and/or getup time. However, getup time was not a sleep parameter included within the parent-reported questionnaire data, and thus we were not able to study this specific sleep timing parameter or to calculate the midpoint of sleep in the whole sample of this study. However, this is something to consider for future research on the topic. Finally, these results were solely conducted in Finnish population. Considering the unique genetic heritage in Finland, ${ }^{54}$ this study should be replicated in other geographical regions. It is, however, noteworthy that the PRSes used in this study were obtained from UK Biobank and 23andMe comprising mostly individuals with European descent. The polygenic risk score derived from UK Biobank ( $\mathrm{PRS}_{\text {BestFit }}$ ) was based on information from SNPs of 301,143 individuals, which gave us a chance to test the best fit p-value threshold in an independent Finnish population sample. PRS ${ }_{10 k B e s t}$ was derived from a larger GWAS containing almost 700,000 individuals from the UK Biobank and 23andMe dataset but with information of only the 10,000 most significant variants. Thus, no populationspecific threshold in the Finnish sample could be calculated.

\section{Conclusion}

PRS for eveningness and morningness are related to sleeponset latency in early childhood. Furthermore, PRS for eveningness associates with later bedtime and to actigraphy-based lowered sleep efficiency. These findings add to the recent research on parental-reported chronotype and children's sleep development, indicating that genetic background for diurnal preference contributes to sleep-wake development in early childhood. Further studies in later stages of childhood should be conducted, to understand whether this genetic influence mainly appears at early stages of life or whether it extends to later stages. Our study helps to clarify the physiological architecture of sleeping in early childhood and may eventually contribute to the development of personalized therapeutic approaches targeting the circadian system.

\section{Acknowledgments}

The authors would like to thank all the families that participated in the CHILD-SLEEP and FinnBrain birth cohorts. The authors are also grateful for the nurses at the maternity clinics who introduced the study to the families.

\section{Funding}

This work was supported by The Academy of Finland (\#134880 and \#253346 to TP; \#308588 to EJP, \#308589 to LK; \# 134950 and \#253270 to HK; \#277557 to OSH; \#315035 to IMM; and \#326631 to AK), the Signe and Ane Gyllenberg foundation, the Yrjö Jahnsson Foundation, the Foundation for Pediatric Research, the Finnish Cultural Foundation, the Competitive Research Financing of the Expert Responsibility area of Tampere University Hospital, the Arvo ja Lea Ylppö Foundation, Finnish 
State Grants for Clinical Research (ERVA), and The Medical Society of Tampere.

\section{Disclosure}

The authors report no conflicts of interest in this work.

\section{References}

1. Kalmbach DA, Schneider LD, Cheung J, et al. Genetic basis of chronotype in humans: insights from three landmark GWAS. Sleep. 2017;40:2. doi:10.1093/sleep/zsw048

2. Güldür TOH. Circadian rhythm in mammals: time to eat \& time to sleep. Biol Rhythm Res. 2017;48(2):243-261. doi:10.1080/ 09291016.2016.1251968

3. Adan A, Archer SN, Hidalgo MP, Di Milia L, Natale V, Randler C. Circadian typology: a comprehensive review. Chronobiol Int 2012;29(9):1153-1175. doi:10.3109/07420528.2012.719971

4. Rijo-Ferreira F, Takahashi JS. Genomics of circadian rhythms in health and disease. Genome Med. 2019;11(1):82. doi:10.1186/ s13073-019-0704-0

5. Cox KH, Takahashi JS. Circadian clock genes and the transcriptional architecture of the clock mechanism. J Mol Endocrinol. 2019;63(4): R93-R102. doi:10.1530/JME-19-0153

6. Barclay NL, Eley TC, Buysse DJ, Rijsdijk FV, Gregory AM. Genetic and environmental influences on different components of the pittsburgh sleep quality index and their overlap. Sleep. 2010;33 (5):659-668. doi:10.1093/sleep/33.5.659

7. Vink JM, Groot AS, Kerkhof GA, Boomsma DI. Genetic analysis of morningness and eveningness. Chronobiol Int. 2001;18(5):809-822. doi:10.1081/CBI-100107516

8. Koskenvuo M, Hublin C, Partinen M, Heikkila K, Kaprio J. Heritability of diurnal type: a nationwide study of 8753 adult twin pairs. J Sleep Res. 2007;16(2):156-162. doi:10.1111/j.1365-2869.2007.00580.x

9. Hu Y, Shmygelska A, Tran D, Eriksson N, Tung JY, Hinds DA. GWAS of 89,283 individuals identifies genetic variants associated with self-reporting of being a morning person. Nat Commun. 2016;7:10448. doi:10.1038/ncomms10448

10. Lane JM, Vlasac I, Anderson SG, et al. Genome-wide association analysis identifies novel loci for chronotype in 100,420 individuals from the UK Biobank. Nat Commun. 2016;7:10889. doi:10.1038/ ncomms 10889

11. Jones SE, Tyrrell J, Wood AR, et al. Genome-wide association analyses in 128,266 individuals identifies new morningness and sleep duration loci. PLoS Genet. 2016;12(8):e1006125. doi:10.1371/ journal.pgen.1006125

12. Jones SE, Lane JM, Wood AR, et al. Genome-wide association analyses of chronotype in 697,828 individuals provides insights into circadian rhythms. Nat Commun. 2019;10(1):343. doi:10.1038/ s41467-018-08259-7

13. Gelbmann G, Kuhn-Natriashvili S, Pazhedath TJ, Ardeljan M, Wober C, Wober-Bingol C. Morningness: protective factor for sleep-related and emotional problems in childhood and adolescence? Chronobiol Int. 2012;29(7):898-910. doi:10.3109/ 07420528.2012.686946

14. Roenneberg T, Allebrandt KV, Merrow M, Vetter C. Social jetlag and obesity. Curr Biol. 2012;22(10):939-943. doi:10.1016/j. cub.2012.03.038

15. Katzenberg D, Young T, Finn L, et al. A CLOCK polymorphism associated with human diurnal preference. Sleep. 1998;21 (6):569-576. doi:10.1093/sleep/21.6.569

16. Lazar AS, Slak A, Lo JC-Y, et al. Sleep, diurnal preference, health, and psychological well-being: a prospective single-allelic-variation study. Chronobiol Int. 2012;29(2):131-146. doi:10.3109/ 07420528.2011 .641193
17. Merikanto I, Paavonen EJ, Saarenpaa-Heikkila O, Paunio T, Partonen T. Eveningness associates with smoking and sleep problems among pregnant women. Chronobiol Int. 2017;34(5):650-658. doi:10.1080/07420528.2017.1293085

18. Simpkin CT, Jenni OG, Carskadon MA, et al. Chronotype is associated with the timing of the circadian clock and sleep in toddlers. J Sleep Res. 2014;23(4):397-405. doi:10.1111/jsr.12142

19. Zimmermann LK. The influence of chronotype in the daily lives of young children. Chronobiol Int. 2016;33(3):268-279. doi:10.3109/ 07420528.2016.1138120

20. Morales-Munoz I, Partonen T, Saarenpaa-Heikkila O, et al. The role of parental circadian preference in the onset of sleep difficulties in early childhood. Sleep Med. 2019;54:223-230. doi:10.1016/j. sleep.2018.10.039

21. Iglowstein I, Jenni OG, Molinari L, Largo RH. Sleep duration from infancy to adolescence: reference values and generational trends. Pediatrics. 2003;111(2):302-307. doi:10.1542/peds.111.2.302

22. Paavonen J, Saarenpää-Heikkilä O, Morales-Munoz I, et al. Normal sleep development in infants: findings from two large birth cohorts. Sleep Med. 2020;69:145-154. doi:10.1016/j.sleep.2020.01.009

23. Petit D, Touchette E, Tremblay RE, Boivin M, Montplaisir J. Dyssomnias and parasomnias in early childhood. Pediatrics. 2007;119(5):e1016-25. doi:10.1542/peds.2006-2132

24. Paavonen J, Saarenpaa-Heikkila O, Polkki P, Kylliainen A, PorkkaHeiskanen T, Paunio T. Maternal and paternal sleep during pregnancy in the Child-sleep birth cohort. Sleep Med. 2017;29:47-56. doi:10.1016/j.sleep.2016.09.011

25. Karlsson L, Tolvanen M, Scheinin NM, et al. Cohort profile: the finnbrain birth cohort study (FinnBrain). Int J Epidemiol. 2018;47 (1):15-16j. doi:10.1093/ije/dyx173

26. Panagiotou OA, Willer CJ, Hirschhorn JN, Ioannidis JPA. The power of meta-analysis in genome-wide association studies. Annu Rev Genomics Hum Genet. 2013;14:441-465. doi:10.1146/annurevgenom-091212-153520

27. Chang CC, Chow CC, Tellier LC, Vattikuti S, Purcell SM, Lee JJ. Second-generation PLINK: rising to the challenge of larger and richer datasets. Gigascience. 2015;4:7. doi:10.1186/s13742-015-0047-8

28. Delaneau O, Zagury J-F, Marchini J. Improved whole-chromosome phasing for disease and population genetic studies. Nat Methods. 2013;10(1):5-6. doi:10.1038/nmeth.2307

29. Howie BN, Donnelly P, Marchini J. A flexible and accurate genotype imputation method for the next generation of genome-wide association studies. PLoS Genet. 2009;5(6):e1000529. doi:10.1371/journal. pgen. 1000529

30. Loh P-R, Danecek P, Palamara PF, et al. Reference-based phasing using the haplotype reference consortium panel. Nat Genet. 2016;48 (11):1443-1448. doi:10.1038/ng.3679

31. Euesden J, Lewis CM, O'Reilly PF. PRSice: polygenic Risk Score software. Bioinformatics. 2015;31(9):1466-1468. doi:10.1093/bioinformatics/btu848

32. Sadeh A. A brief screening questionnaire for infant sleep problems: validation and findings for an Internet sample. Pediatrics. 2004;113 (6): e570-7. doi:10.1542/peds.113.6.e570

33. Morrell JM. The infant sleep questionnaire: a new tool to assess infant sleep problems for clinical and research purposes. Child Psychol Psychiatry Rev. 1999;4:20-26. doi:10.1017/ S1360641798001816

34. Choi BCK, Pak AWP. A catalog of biases in questionnaires. Prev Chronic Dis. 2005;2(1):A13.

35. Romeo DM, Bruni O, Brogna C, et al. Application of the sleep disturbance scale for children (SDSC) in preschool age. Eur J Paediatr Neurol. 2013;17(4):374-382. doi:10.1016/j.ejpn.2012.12.009

36. Bruni O, Ottaviano S, Guidetti V, et al. The Sleep Disturbance Scale for Children (SDSC). Construction and validation of an instrument to evaluate sleep disturbances in childhood and adolescence. $J$ Sleep Res. 1996;5(4):251-261. doi:10.1111/j.1365-2869.1996.00251.x 
37. Tynjälä J, Kannas L, Levälahti E. Perceived sleep quality and its precursors in adolescents. J Sleep Res. 1997;6:189-198. doi:10.1046/ j.1365-2869.1997.00048.x

38. Oakley NR. Validation with Polysomnography of the Sleepwatch Sleep/Wake Scoring Algorithm Used by the Actiwatch Activity Monitoring. Technical Report to Mini Mitter Co., Inc., System; 1997.

39. So K, Buckley P, Adamson TM, Horne RSC. Actigraphy correctly predicts sleep behavior in infants who are younger than six months, when compared with polysomnography. Pediatr Res. 2005;58 (4):761-765. doi:10.1203/01.PDR.0000180568.97221.56

40. Meltzer LJ, Montgomery-Downs HE, Insana SP, Walsh CM. Use of actigraphy for assessment in pediatric sleep research. Sleep Med Rev. 2012;16(5):463-475. doi:10.1016/j.smrv.2011.10.002

41. Benjamini Y, Hochberg Y. Controlling the false discovery rate: a practical and powerful approach to multiple testing. $J R$ Stat Soc Ser B. 1995;57(1):289-300.

42. $\mathrm{R}$ Core Team. $R:$ A Language and Environment for Statistical Computing. Vienna: R Foundation for Statistical Computing; 2019.

43. Schwarzer G. meta: an R Package for Meta-Analysis. R News. 2007;7 (3):40-45.

44. Sletten TL, Rajaratnam SMW, Wright MJ, et al. Genetic and environmental contributions to sleep-wake behavior in 12-year-old twins. Sleep. 2013;36(11):1715-1722. doi:10.5665/sleep.3136

45. Kushnir J, Sadeh A. Correspondence between reported and actigraphic sleep measures in preschool children: the role of a clinical context. J Clin Sleep Med JCSM off Publ Am Acad Sleep Med. 2013;9(11):1147-1151. doi:10.5664/jcsm.3154

46. Goodlin-Jones BL, Tang K, Liu J, Anders TF. Sleep patterns in preschool-age children with autism, developmental delay, and typical development. J Am Acad Child Adolesc Psychiatry. 2008;47 (8):930-938. doi:10.1097/CHI.ObO13e3181799f7c
47. Jafar NK, Tham EKH, Eng DZH, et al. The association between chronotype and sleep problems in preschool children. Sleep Med. 2017;30:240-244. doi:10.1016/j.sleep.2016.11.015

48. Gedda L, Brenci G. Sleep and dream characteristics in twins. Acta Genet Med Gemellol. 1979;28(3):237-239. doi:10.1017/ s0001566000009089

49. Gregory AM, Rijsdijk FV, Eley TC. A twin-study of sleep difficulties in school-aged children. Child Dev. 2006;77(6):1668-1679. doi:10.1111/j.1467-8624.2006.00966.x

50. Dashti HS, Jones SE, Wood AR, et al. Genome-wide association study identifies genetic loci for self-reported habitual sleep duration supported by accelerometer-derived estimates. Nat Commun. 2019;10 (1):1100. doi:10.1038/s41467-019-08917-4

51. Brescianini S, Volzone A, Fagnani C, et al. Genetic and environmental factors shape infant sleep patterns: a study of 18-month-old twins. Pediatrics. 2011;127(5):e1296-302. doi:10.1542/peds.20100858

52. Swaminathan K, Klerman EB, Phillips AJK. Are individual differences in sleep and circadian timing amplified by use of artificial light sources? J Biol Rhythms. 2017;32(2):165-176. doi:10.1177/ 0748730417699310

53. Morales-Munoz I, Saarenpaa-Heikkila O, Kylliainen A, et al. The effects of maternal risk factors during pregnancy on the onset of sleep difficulties in infants at 3 months old. J Sleep Res. 2018;27(5): e12696. doi:10.1111/jsr.12696

54. Kääriäinen H, Muilu J, Perola M, Kristiansson K. Genetics in an isolated population like Finland: a different basis for genomic medicine? J Community Genet. 2017;8(4):319-326. doi:10.1007/ s12687-017-0318-4

\section{Publish your work in this journal}

Nature and Science of Sleep is an international, peer-reviewed, open access journal covering all aspects of sleep science and sleep medicine, including the neurophysiology and functions of sleep, the genetics of sleep, sleep and society, biological rhythms, dreaming, sleep disorders and therapy, and strategies to optimize healthy sleep.
The manuscript management system is completely online and includes a very quick and fair peer-review system, which is all easy to use. Visit http://www.dovepress.com/testimonials.php to read real quotes from published authors. 